\title{
2-YIA-41 YIA
}

\section{Effects of Goreisan on LPS-induced diarrhea and decrease in aquaporin 3 expression in intestinal epithelial cells.}

\author{
Kazuhito Murakami, Shingo Matsuyama, Yoichiro Isohama
}

Lab. Appl. Pharmacol., Facul. of Pharm. Sci., Tokyo Univ. of Sci.

Goreisan is often used for gastrointestinal symptoms associated with bacterial and viral infections, to care diarrhea and to prevent general dehydration. Although several clinical reports have shown the effectiveness of Goreisan, pharmacological properties and underlining mechanism of Goreisan has not been clear. In this study, therefore, we investigated the antidiarrheic effect of Goreisan using a mouse model of enterocolitis induced by Lipopolysaccharide (LPS). Goreisan did not affect TNF- $\alpha$ mRNA expression, but markedly improved tissue injury and diarrhea scores. On the other hand, aquaporin-3 (AQP3) is expressed in the intestinal epithelium, and responsible for the absorption of water in the intestinal tract. Interestingly, both AQP3 mRNA and protein expression in the intestinal epithelium in LPS-treated group were significantly reduced, and Goreisan inhibited this decrease in AQP3. Decrease in AQP3 is thought to be associated with development of diarrhea, and therefore, Goreisan is estimated to have improved diarrhea symptoms by regulating the expression of AQP3. These results confirmed the effectiveness of Goreisan for infectious gastroenteritis, and it is also suggested a new effect of Goreisan. 